\title{
Immune cells in the small intestinal mucosa of newborn yaks
}

\author{
Q. Zhang ${ }^{1}$, Y. Cui ${ }^{1}$, S.J. Yu², Y.F. Huang ${ }^{1}$, Y.Y. Pan², Z.C. Bai ${ }^{1}$ \\ ${ }^{1}$ College of Veterinary Medicine, Gansu Agricultural University, Lanzhou, Gansu, China \\ ${ }^{2}$ Gansu Province Livestock Embryo Engineering Research Centre, College of Veterinary Medicine, \\ Gansu Agricultural University, Lanzhou, Gansu, China \\ [Received: 16 June 2021; Accepted: 8 September 2021; Early publication date: 7 October 2021]
}

Background: This study aimed to characterise and evaluate the main markers of T lymphocytes, B lymphocytes, immunoglobulin (Ig) A and IgG plasmocytes, macrophages, and dendritic cells of the intestinal mucosa of newborn yaks.

Materials and methods: Ten newborn yaks (2-4 weeks old) were chosen. Immunohistochemistry and real-time quantitative polymerase chain reaction were used to analyse the immune cell distribution and specific markers at the mRNA expression level in the duodenum, jejunum, and ileum.

Results: The results showed in the epithelium, CD3e-positive $T$ lymphocyte levels were higher than other immune cell levels $(p<0.05)$. Additionally, in the lamina propria, the number of cells positive for $C D 3 \varepsilon, C D 68$, and signal inhibitory regulatory protein alpha (SIRP $\alpha$ ) were higher in the villi, while CD79 $\alpha, \lg A$ and IgG cells were more common at the base of the crypt. Moreover, both in the epithelium and lamina propria, the number of CD3E, CD68 and SIRP $\alpha$ were decreased from the duodenum to the ileum ( $p<0.05$ ), additionally the number of $C D 79 \alpha, \lg A$ and $\lg G$ positive cells were increased from the duodenum to the ileum of newborn yaks $(p<0.05)$. Furthermore, the mRNA expression levels of $C D 3 \varepsilon, C D 68$, and SIRPa increased from the duodenum to the ileum $(p<0.05)$, while the mRNA expression levels of CD79 $\alpha, \lg A$ and $\lg G$ decreased from the duodenum to the ileum.

Conclusions: Immunohistochemical characterisation and expression levels of immune factors in the small intestinal mucosa of newborn yaks suggest that the intestinal mucosa is an important part of the natural barrier and provides useful references for immunity functions of newborn yak intestinal mucosa. (Folia Morphol 2022; 81, 1: 91-100)

Key words: newborn yaks, small intestine, mucosa, immunity cell

\section{INTRODUCTION}

Domesticated yaks are a valuable breed resource in the Qinghai-Tibet Plateau. They have unique ecological characteristics and are valued for their meat, draft and milk [19]. Highland pastoral areas are cur- rently dominated by traditional grazing yak breeding; however, due to the harsh plateau environment, extensive management, and the influence of pathogenic factors, the intestinal mucosal immune barrier of yaks is highly susceptible to damage. Therefore,

Address for correspondence: Prof. Y. Cui, Faculty of Veterinary Medicine, Gansu Agricultural University, Lanzhou, Gansu, China, fax: +86-0931-7631220, e-mail: cuiyan369@sina.com

This article is available in open access under Creative Common Attribution-Non-Commercial-No Derivatives 4.0 International (CC BY-NC-ND 4.0) license, allowing to download articles and share them with others as long as they credit the authors and the publisher, but without permission to change them in any way or use them commercially. 
the incidence of gastrointestinal function disorder, inflammatory bowel disease, and infectious diarrhoea disease is high. These illnesses increase the length of the growth cycle and, as a result, the yak develops slowly. The mortality rate of newborn yaks is as high as $30 \%$ [9], which causes serious damage to the development of agriculture. Therefore, in order to improve yak survival and productivity, it is imperative to understand the characteristics of the small intestinal mucosal immune cells of yaks.

The small intestinal tract contains the largest number of immune cells, comprising a heterogeneous population of $T$ and $B$ lymphocytes, plasmocytes, macrophages, dendritic cells and a variety of non-professional antigen-presenting cells $[8,18]$. Appropriate interactions between these different cell types are essential for generating immune responsiveness or tolerance to a large array of environmental antigens. The previous study analysed the distribution and population of immunocompetent cells in the small intestine of sheep, pigs, calves and mice $[4,6,21,22]$. However, due to the limitation of the global yak distribution, there are few reports on the immune cells of yaks.

The aim of the present study was to provide basic data on the characteristics of immune cells and factors in the small intestine of healthy newborn yaks. We used CD3 $\varepsilon, C D 79 \alpha$, immunoglobulin (Ig) A, IgG, CD68, and signal inhibitory regulatory protein alpha (SIRP $\alpha$ ) to characterise T and B lymphocytes, plasmocytes, macrophages, and dendritic cells in the small intestine of newborn yaks, and assayed the mRNA expression levels of immune cell-specific markers.

\section{MATERIALS AND METHODS}

\section{Animals and tissues collection}

All experimental animals were handled according to the Animal Ethics Procedures and Guidelines of the People's Republic of China and were approved by the Institutional Animal Care and Use Committee of the College of Veterinary Medicine of Gansu Agricultural University. All yaks were considered healthy based on the results of physical examination and serum biochemical analysis. The animals were euthanased with an intravenous injection of pentobarbital sodium $(200 \mathrm{mg} / \mathrm{kg})$. To maintain the original habitat of the animals, the yaks were sacrificed, and samples were collected from local farms.

Ten newborn yaks (2-4 weeks old) were obtained on a local farmer in Xining City, Qinghai Province. The small intestinal regions (duodenum, jejunum, and ileum) were excised from each animal and samples were taken for immunohistochemical and polymerase chain reaction (PCR) analysis. All specimens intended for immunohistochemical analysis were fixed in $4 \%$ neutral paraformaldehyde phosphate buffer ( $\mathrm{pH}$ 7.3). Specimens intended for real-time quantitative PCR (RT-qPCR) were flash frozen and stored in liquid nitrogen until further processing.

\section{Relative RT-qPCR}

Total RNA was isolated from the small intestine using TRIzol reagent (Invitrogen, Carlsbad, CA, USA). RNA was reverse transcribed to single-stranded CDNA using a reverse transcription kit (MBI Fermentas, Burlington, ON, Canada) according to the manufacturer's instructions. The RT-qPCR primers were designed according to the Bos grunniens CD3 $\varepsilon, C D 79 \alpha$, $\lg \mathrm{A}, \lg \mathrm{G}, \mathrm{CD} 68, \operatorname{SIRP} \alpha$, and $\beta$-actin gene sequences (GenBank accession numbers: KY911279, KY911280, MG432919, MF099643, KY921959, MH347358, and DQ838049, respectively) using Primer 5 software and synthesised by the Beijing Genomics Institute BGI Company (China). The RT-qPCR primer sequences are presented in Table 1. RT-qPCR was conducted using a Light-Cycler480 thermocycler (Roche, Manheim, Germany) in 20- $\mu \mathrm{L}$ reaction volumes consisting of $1 \mu \mathrm{L} \mathrm{cDNA}, 1 \mu \mathrm{L}$ forward primer, $1 \mu \mathrm{L}$ reverse primer, $10 \mu \mathrm{L} 2 \times$ SYBR Green II PCR Master Mix (TaKaRa, Shiga, Japan), $0.4 \mu \mathrm{L}$ Rox, and $6.4 \mu \mathrm{L}$ nuclease-free $\mathrm{H}_{2} \mathrm{O}$. Four replicates were set for each sample to ensure the accuracy of the relative expression of the target genes in the sample. After amplification, according to the system-generated $\mathrm{Ct}$ value, the $2^{-\Delta \Delta C t}$ method was used with $\beta$-actin as an internal standard to obtain the relative expression of $\mathrm{CD} 3 \varepsilon, \mathrm{CD} 79 \alpha$, IgA, IgG, CD68 and SIRP $\alpha$ mRNA.

\section{Immunohistochemical examination}

The spatial distribution of cells positive for $\mathrm{CD} 3 \varepsilon$, CD79 $\alpha, \lg A, \lg G, C D 68$ and SIRP $\alpha$ in the small intestine of newborn yaks was evaluated by immunohistochemical staining. Fixed tissue sections were mounted on microscope slides in a routine manner and exposed to primary antibodies against $\mathrm{CD} 3 \varepsilon$ (monoclonal rabbit anti-cow CD3e, Abcam, Cambridge, UK; ab16669, 1:200 dilution), $C D 79 \alpha$ (monoclonal mouse anti-cow CD79 $\alpha$, Abcam; ab199001, 1:100 dilution), IgA (polyclonal rabbit anti-cow IgA, Abcam; ab112630, 1:100 dilution), IgG (polyclonal rabbit anti-cow IgG, Abcam; ab6692, 1:100 dilution), SIRP $\alpha$ (polyclonal rabbit an- 
Table 1. Primers used in this study

\begin{tabular}{|c|c|c|c|c|}
\hline Genes & Primer names & Primer sequences $\left(5^{\prime}-3^{\prime}\right)$ & Length (bp) & Annealing $\left({ }^{\circ} \mathrm{C}\right)$ \\
\hline \multirow[t]{2}{*}{$\operatorname{CD} 3 \varepsilon$} & P1 & F: GGGCTCATAGTCTGGATTGG & 155 & 57 \\
\hline & P2 & R: TGTGTCACTCTGGGCTTGC & & \\
\hline \multirow[t]{2}{*}{$\operatorname{CD79} \alpha$} & P3 & F: ACGGCAAGAAGATTCAGCG & 182 & 60 \\
\hline & P4 & R: CCAAGGAGGCAATAGGAG & & \\
\hline \multirow[t]{2}{*}{$\lg A$} & P5 & F: GGTTCACAGGACCCAGA & 227 & 57 \\
\hline & P6 & R: AGCACCTAGTGAAGCCC & & \\
\hline \multirow[t]{2}{*}{$\lg G$} & P7 & F: AACCAACACCACAGGAAC & 208 & 60 \\
\hline & P8 & R: AGTGTAGTCTCCTATTGCCT & & \\
\hline \multirow[t]{2}{*}{ CD68 } & P9 & F: TGAGAGGAGCAAGTGGGA & 194 & 56 \\
\hline & P10 & R: GTGGACATCATCCTGGCTGG & & \\
\hline \multirow[t]{2}{*}{$\operatorname{SIRP} \alpha$} & P11 & F: ATCCTGCTGCCCGCTGTA & 215 & 60 \\
\hline & $\mathrm{P} 12$ & R: AACAGTTGGGCGGCGAG & & \\
\hline \multirow[t]{2}{*}{$\beta$-actin } & $\mathrm{P} 13$ & F: AGGCTGTGCTGTCCCTGTATG & 207 & 60 \\
\hline & P14 & R: GCTCGGCTGTGGTGGTAAA & & \\
\hline
\end{tabular}

ti-cow SIRP $\alpha$, Abcam; ab116254, 1:100 dilution), and CD68 (polyclonal rabbit anti-cow CD68, Abbiotec, San Diego, USA; No:252281, 1:100 dilution) for $2 \mathrm{~h}$ at $37^{\circ} \mathrm{C}$ in a moist chamber. Biotinylated secondary antibodies were applied for $10 \mathrm{~min}$. Streptavidin-conjugated peroxidase was then applied to the slides for $10 \mathrm{~min}$. The reaction products were formed using 3,3-diaminobenzidine tetrahydrochloride. The sections were lightly counterstained with haematoxylin. The negative control for each sample was created by replacing the primary antibody with serum albumin; all other steps and conditions remained the same.

\section{Examination of sections}

The sections were examined under a light microscope with image analysis software (Image-Pro Plus, Media Cybemetics, Silver Spring, MD, USA). Cells positive for $\mathrm{CD} 3 \varepsilon, \mathrm{CD} 79 \alpha, \lg A, \lg G, \operatorname{SIRP} \alpha$ and CD68 in the small intestine of newborn yaks were evaluated. Subjective analysis of the distribution of positively labelled cells in the lamina propria and epithelial compartments was performed using a $\times 40$ objective.

In each tissue sample, the number of positively labelled cells was determined in three standard areas (Fig. 1) of the lamina propria: the villi, the upper crypt, and the base of the crypt. Five images were randomly chosen for each of these areas. Results were expressed as cells per $10000 \mu \mathrm{m}^{2}$ of lamina propria tissue [7]. Intraepithelial immune cells were assessed by counting positively labelled cells in five areas (each of 100 enterocytes) of the epithelium, and the results were

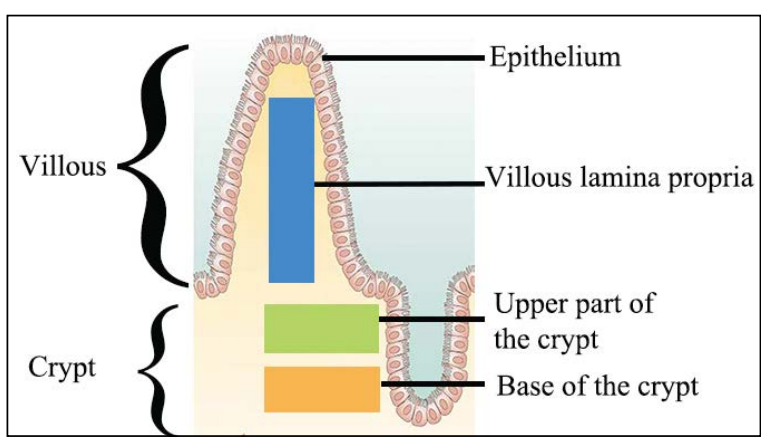

Figure 1. Diagram of small intestinal epithelium and lamina propria to demonstrate the areas used for counting.

expressed as the mean number of cells/100 enterocytes. Repeated independent counts were performed on five serial sections from the same tissue block to assess the precision of both the lamina propria and epithelial cell counting techniques.

\section{Statistical analysis}

All statistical analyses were performed using SPSS (version 21.0; IBM Corp., Armonk, NY, USA). The relative mRNA levels and positive cell numbers among the study groups are expressed as mean \pm standard error. Statistical significance was determined using one-way analysis of variance and was set at $p<0.05$.

\section{RESULTS}

Morphological analysis of the crypt-villus axis

The small intestinal mucosa of newborn yaks can be divided into three layers: the epithelium, lamina 

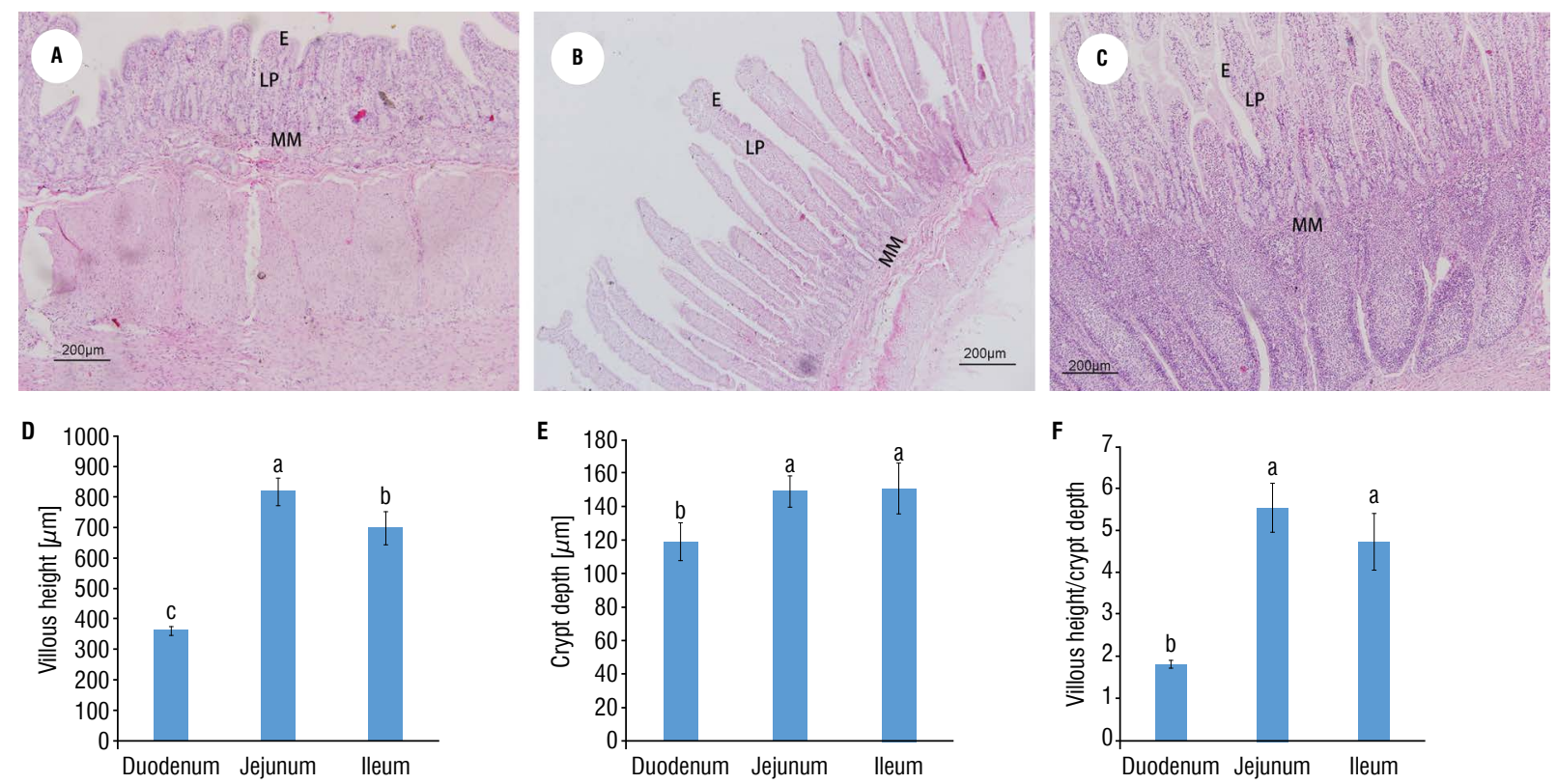

Figure 2. Morphological characteristics and analysis (including villous height, crypt depth, and ratio of villous height to crypt depth) of the small intestinal mucosa in newborn yaks; A-C. The histological section of the duodenum, jejunum and ileum in newborn yaks $(\mathrm{HE}, \times 100)$; D. The villous height of the duodenum, jejunum and ileum in newborn yaks; $\mathbf{E}$. The crypt depth of the duodenum, jejunum and ileum in newborn yaks; F. Ratio of villous height to crypt depth of the duodenum, jejunum and ileum in newborn yaks. Bars with different superscripts are significantly different $(p<0.05)$. The results were presented as mean \pm standard error; $\mathrm{E}-$ mucosal epithelium; $\mathrm{LP}$ - lamina propria; $\mathrm{MM}$ - muscularis mucosa.

propria and mucosal muscle. The morphological parameters of the crypt-villus axis in the small intestine include villous height, crypt depth, and the ratio of villous height to crypt depth (Fig. 2A-C). The villous height was highest in the jejunum, followed by the ileum and the duodenum ( $p<0.05$ ) (Fig. 2D). Crypt depth was higher in the jejunum and ileum than in the duodenum ( $p<0.05$ ) (Fig. 2E). Moreover, there were no differences between the jejunum and ileum $(p>0.05)$. Furthermore, the ratio of villous height to crypt depth was higher in the jejunum and ileum than in the duodenum ( $p<0.05)$ (Fig. 2F).

\section{$\mathrm{CD} 3 \varepsilon, \mathrm{CD} 79 \alpha, \lg \mathrm{A}, \lg \mathrm{G}, \mathrm{CD} 68$ and $\operatorname{SIRP} \alpha$ mRNA expression}

The relative expression levels of $\mathrm{CD} 3 \varepsilon, \mathrm{CD} 79 \alpha, \lg \mathrm{G}$, IgA, CD68 and SIRP $\alpha$ mRNA differed between the duodenum, jejunum and ileum of newborn yaks (Fig. 3). Within the same intestinal region, the expression levels of $\mathrm{CD} 3 \varepsilon, \mathrm{CD} 68$ and SIRP $\alpha$ mRNA were significantly higher than those of $\mathrm{CD} 79 \alpha, \lg A$ and $\operatorname{lgG}$. Additionally, in the different intestinal regions, $C D 3 \varepsilon, C D 68$ and SIRP $\alpha$ mRNA expression levels increased from the duodenum to the ileum $(p<0.05)$, while the mRNA expression levels of $C D 79 \alpha, \lg A$ and $\lg G$ decreased from the duodenum to the ileum.

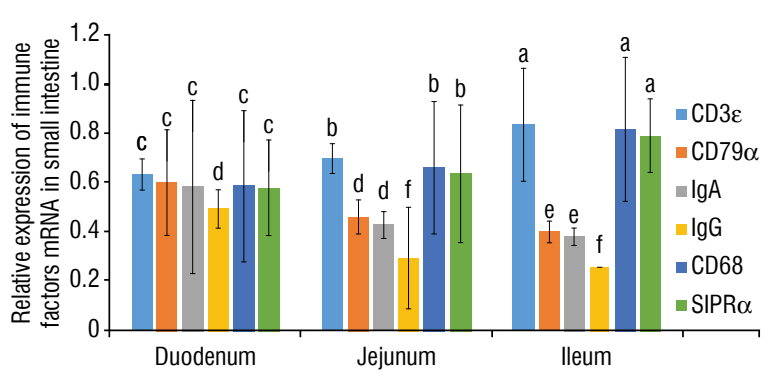

Figure 3. Relative abundance of $\operatorname{CD} 3 \varepsilon, \operatorname{CD} 79 \alpha$, immunoglobulin A $(\lg A)$, immunoglobulin $\mathrm{G}(\lg \mathrm{G}), \mathrm{CD} 68$ and signal inhibitory regulatory protein alpha (SIRP $\alpha$ ) mRNA in the duodenum, jejunum and ileum of newborn yaks. Bars with different superscripts are significantly different $(p<0.05)$. Data are normalised and presented as ratio mean \pm standard error, with $\beta$-actin as an internal control.

\section{CD3 $\varepsilon$-positive T lymphocytes in the small intestinal mucosa}

The membrane staining of $C D 3 \varepsilon$-positive cells was located in the epithelium and lamina propria of the small intestinal mucosa of newborn yaks (Fig. 4A-C). In the epithelium, the number of $\mathrm{CD} 3 \varepsilon$-positive $T$ lymphocytes increased from the duodenum to the ileum, peaking at the ileum ( $p<0.05$ ) (Fig. 5). The difference between the duodenum and jejunum was not significant ( $p>0.05$ ). In the lamina propria, CD3-positive T lymphocytes increased from the basal 

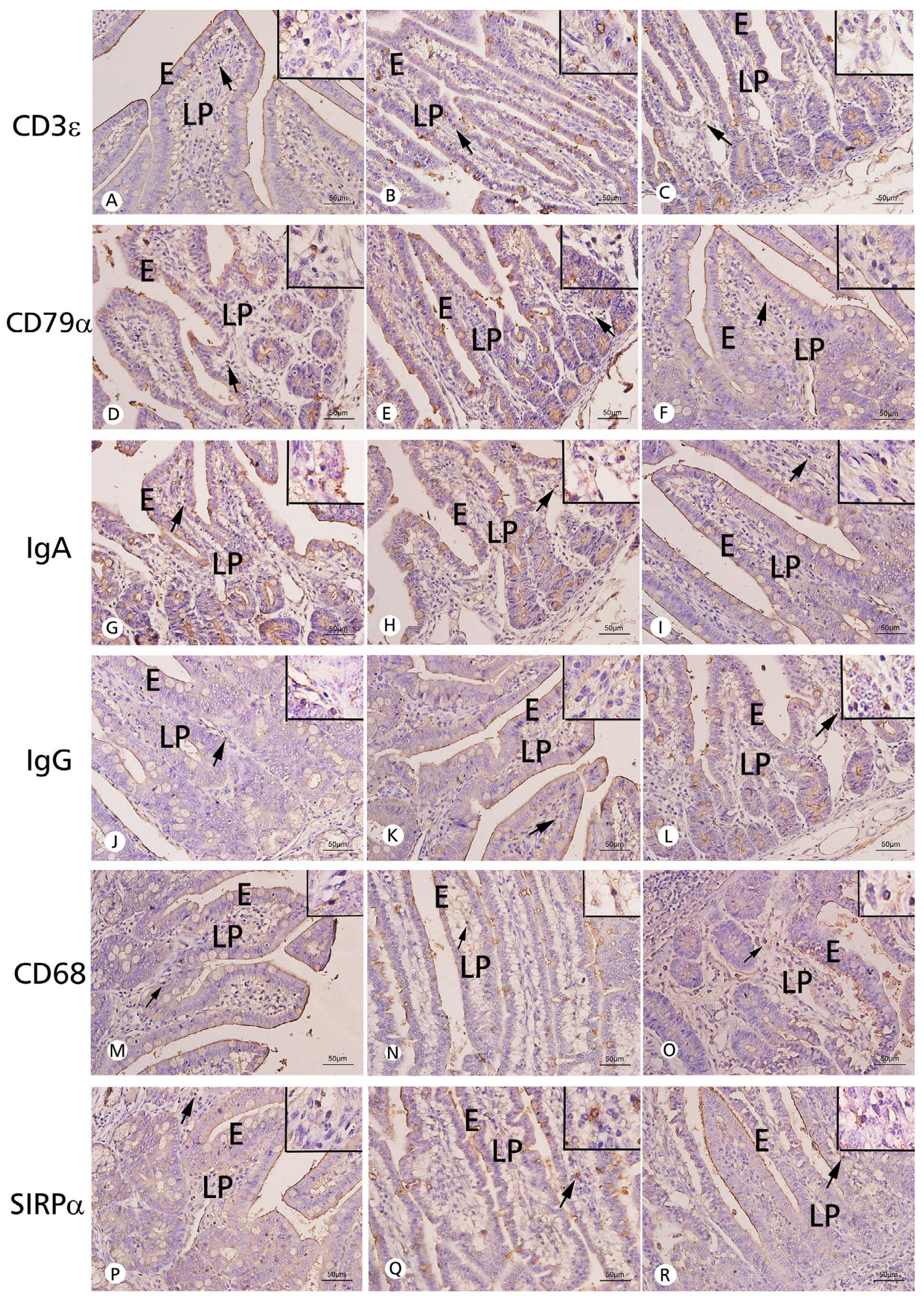

Figure 4. A-R. Immunohistochemical staining of cells expressing CD3ع, CD79 $\alpha$, immunoglobulin A (IgA), immunoglobulin G (IgG), CD68 and signal inhibitory regulatory protein alpha $(\operatorname{SIRP} \alpha)$ in the duodenum, jejunum and ileum of newborn yaks; $\mathrm{E}$ - mucosal epithelium; $\mathrm{LP}$ - lamina propria. Arrows show the positive cells (brown). Bar $=50 \mu \mathrm{m}$ (low-power lens), bar $=20 \mu \mathrm{m}$ (high-power lens on the upper right). 


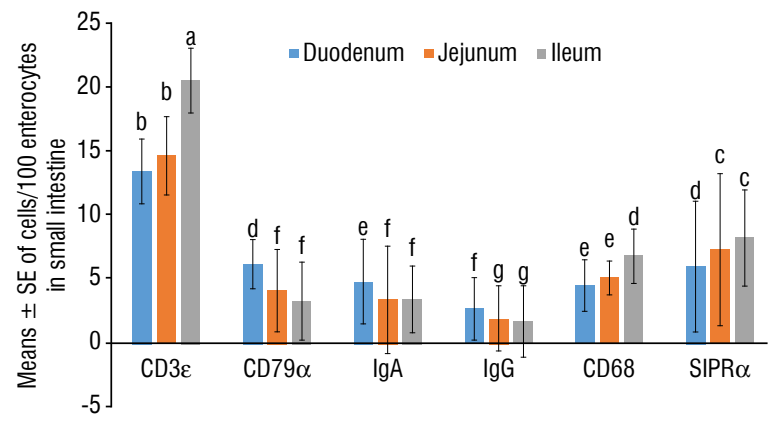

Figure 5. The number of $\mathrm{CD} 3 \varepsilon, \mathrm{CD} 79 \alpha$, immunoglobulin $\mathrm{A}(\lg \mathrm{A})$, immunoglobulin $\mathrm{G}(\mathrm{IgG}), \mathrm{CD} 68$ and signal inhibitory regulatory protein alpha (SIRP $\alpha$ ) positive cells in mucosal epithelium from the duodenum, jejunum and ileum of newborn yaks. Bars with different superscripts are significantly different $(p<0.05)$. Data are normalised and presented as mean \pm standard error (SE) of cells/ $/ 100$ enterocytes.

crypt to the villi (Fig. 6A). Additionally, the number of CD3-positive cells in the lamina propria was higher in the ileum than in the duodenum and jejunum.

\section{CD79 $\alpha$-positive B lymphocytes in the small intestinal mucosa}

The membrane staining of $C D 79 \alpha$-positive B lymphocytes was localised to the epithelium and lamina propria of the small intestinal mucosa of newborn yaks (Fig. 3D-F). CD79 $\alpha$-positive B lymphocyte levels were highest in the epithelium of the duodenum and then decreased from the duodenum to the ileum $(p<0.05)$ (Fig. 5). Additionally, in the lamina propria, CD79 $\alpha$-positive B lymphocytes increased from the villi to the basal crypt. Moreover, the number of CD79 $\alpha$-positive B lymphocytes in the lamina propria was higher in the duodenum than in the ileum and jejunum $(p<0.05)$ (Fig. 6B).

\section{IgA- and IgG-positive plasmocytes in the small intestinal mucosa}

IgA and IgG markers stained the cytoplasm of the plasmocytes. Positive cells appeared in the epithelium and lamina propria of the small intestinal mucosa of newborn yaks (Fig. 3G-L). IgA- and IgG-positive plasmocytes were highest in the epithelium of the duodenum but decreased from the duodenum to the ileum ( $<0.05)$ (Fig. 5). There were no significant differences between the duodenum and the ileum. Furthermore, in the lamina propria, IgA- and IgG-positive plasmocytes increased from the villi to the basal crypt in all regions of the intestine, peaking in the basal crypt $(p<0.05)$ (Fig. 6C, D). Moreover, the number of IgA- and IgG-positive plasmocytes in the lamina propria was higher in the duodenum than in the ileum and jejunum.

\section{CD68-positive macrophages in the small intestinal mucosa}

CD68-positive macrophages presented as large, irregular, or elongated cells scattered in the epithelium and lamina propria of the small intestinal mucosa of newborn yaks (Fig. 3M-O). In the epithelium, CD68-positive macrophages increased from the duodenum to the ileum, peaking in the ileum $(p<0.05)$ (Fig. 5). The difference between the duodenum and jejunum was not significant $(p>0.05)$. Furthermore, in the lamina propria, CD68-positive macrophages increased from the basal crypt to the villi in all regions of the intestine, peaking in the villi $(p<0.05)$ (Fig. 6E). Additionally, the number of $\mathrm{CD} 68$ positive macrophages in the lamina propria was higher in the ileum than in the duodenum and jejunum $(p<0.05)$.

\section{SIRP $\alpha$-positive dendritic cells in the small intestinal mucosa}

Strong SIRP $\alpha$ cytoplasmic staining of dendritic cells was observed in the epithelium and lamina propria of the small intestinal mucosa of newborn yaks (Fig. 3P-R). SIRP $\alpha$-positive dendritic cell numbers were higher in the epithelium of the ileum and jejunum than in the epithelium of the duodenum $(p<0.05)$ (Fig. 5). Furthermore, in the lamina propria, SIRP $\alpha$-positive dendritic cells increased from the basal crypt to the villi, peaking in the villi $(p<0.05)$ (Fig. 6F). Moreover, the number of SIRP $\alpha$-positive dendritic cells in the lamina propria was higher in the ileum than in the duodenum and jejunum.

\section{DISCUSSION}

Many studies have focused on immunocompetent cells in various species, including lambs [4], calves [6], pigs [21], and mice [22]. However, there are limited data available on the small intestine of newborn yaks. To our knowledge, this is the first study to investigate the distribution of immunocompetent cells of the mucosa and to characterise the changes in these cell markers in the small intestine of newborn yak.

The inner surface of the small intestine is covered with finger-like projections called villi that increase the surface area available for the absorption of nutrients from the gut content. The villi increase the length of 

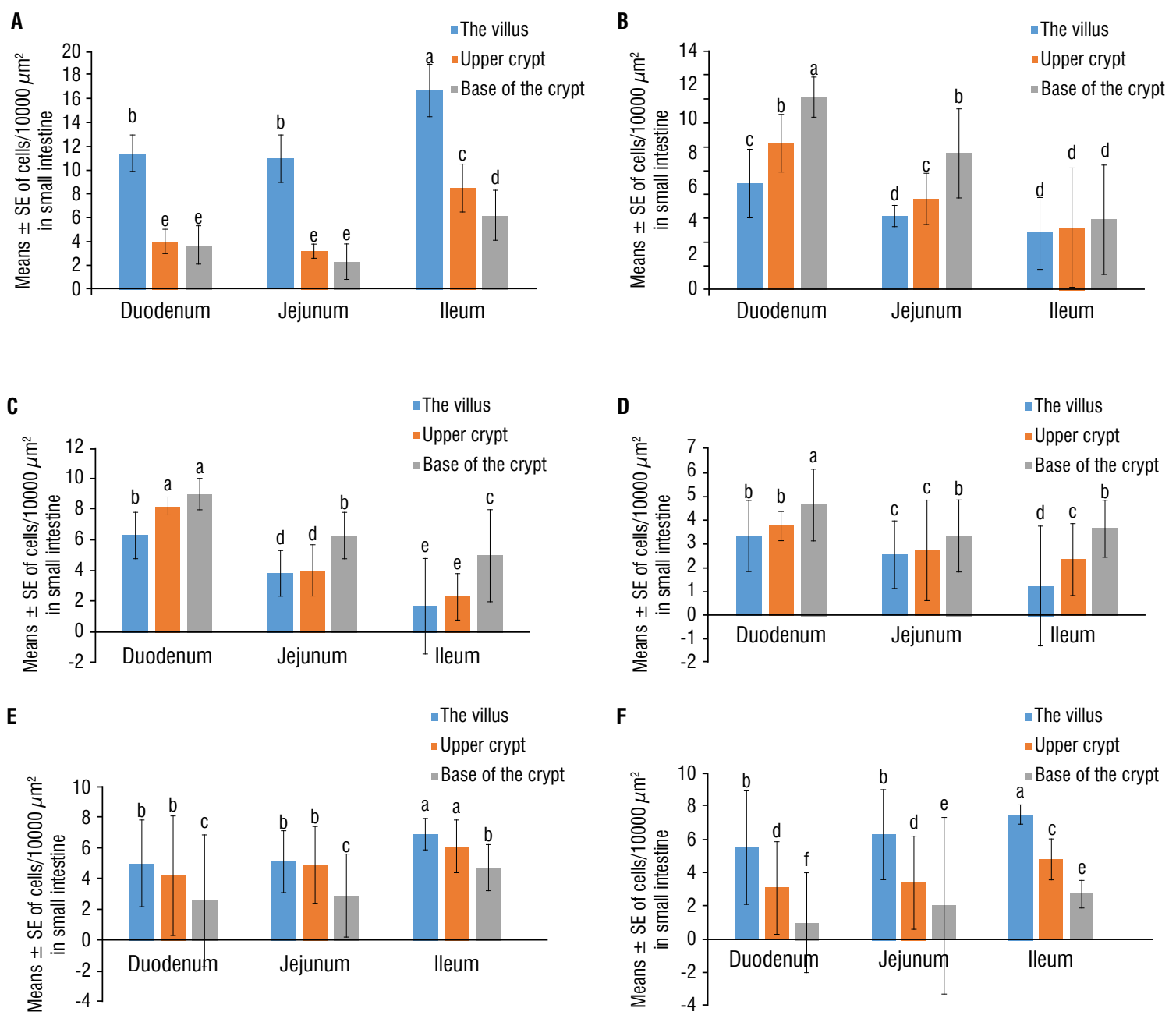

Figure 6. The number of $\operatorname{CD} 3 \varepsilon(\mathbf{A}), \operatorname{CD79} \alpha(\mathbf{B})$, immunoglobulin $A(\lg A)(\mathbf{C})$, immunoglobulin $\mathrm{G}(\lg G)(\mathbf{D}), \operatorname{CD68}(\mathbf{E})$ and signal inhibitory regulatory protein alpha $(\operatorname{SIRP} \alpha)(\mathbf{F})$ positive cells in mucosal lamina propria from duodenum, jejunum and ileum of newborn yaks. Bars with different superscripts are significantly different $(\mathrm{p}<0.05)$. Data are normalised and presented as mean \pm standard error $(\mathrm{SE})$ of cells $/ 10000 \mu \mathrm{m}^{2}$.

the small intestine and therefore increase the likelihood of a food particle encountering a digestive enzyme and being absorbed across the epithelium and into the bloodstream [13]. In the present study, the largest villous height was observed in the jejunum of newborn yaks; thus, the highest rate of absorption may occur in the jejunum. On the other hand, Zhou [28] reported crypts are formed by secretory epithelial cells. In this study, the deepest crypt depths were located in the jejunum and ileum of the newborn yaks, indicating the highest rate of digestion occurs in the jejunum and ileum of newborn yaks. Furthermore, the ratio of villous height to crypt depth reflected the functional state of the small intestine, with a high ratio indicating a high elimination and absorption function [28]. We also found that the ratio of villous height to crypt depth was higher in the jejunum and ileum than in the duodenum. This may indicate that the highest rate of absorption and digestion occurs in the jejunum and ileum of newborn yaks.

Possessing the characteristics of both innate and adaptive immunity, T lymphocytes in the mucosa serve as an effective first-line defence against invasive microorganisms [23]. CD3 $\varepsilon$ is an important differentiation antigen on the surface of the $T$ lymphocyte membrane and a characteristic marker of mature T lymphocytes [10]. We found that the number of $\mathrm{CD} 3 \varepsilon$-positive $T$ lymphocytes was higher in the epithelium and lamina propria of the ileum than those of the duodenum. Consistently, in cats, calves, and goats, the total number of $\mathrm{T}$ lymphocytes is greatest in the ileum $[4,6,24]$. Ma reported that $C D 3 \varepsilon$-positive $\mathrm{T}$ lymphocytes in the mucosa of the small intestine can rapidly respond to microbial invasion by activating host defence responses, including the production of mucus and antimicrobial peptides, which 
help prevent microbes from reaching the epithelial surface [14]. Additionally, during active infection, T lymphocytes in the mucosa promote epithelial cytolysis and cytokine and chemokine production, which serve to limit pathogen invasion, replication, and dissemination [17]. The distribution characteristics of CD3e-positive T lymphocytes in the intestinal mucosa in this study suggest that cellular immunity of the intestinal mucosa of newborn yaks mainly occurs in the ileum. Furthermore, we found that the number of CD3E-positive cells in the lamina propria was higher in the villi than in either of the two crypt regions in newborn yaks. The increase in T lymphocyte density towards the villous tip likely reflects the increased exposure to luminal antigens at this site.

$C D 79 \alpha$ is a common marker of $B$ lymphocytes that plays a key role in $B$ lymphocyte antigen receptor signal transduction, development, stabilisation, and function [20]. In the present study, a significant number of $C D 79 \alpha$-positive cells were observed in the basal crypt area of the lamina propria. Wang reported that B cells of the lamina propria have an increased expression of surface activation markers and exhibit spontaneous immunoglobulin secretion [25]. This indicates that the basal crypt area may be the site of $B$ cell terminal differentiation in the development of immune responses against intestinal antigens. The present study showed a higher number of B lymphocytes in the duodenal epithelium and lamina propria of newborn yaks than in the ileum; similar results have been obtained in lambs and calves $[4,6]$. We speculated that the duodenum is likely the first site to come into contact with foreign antigens and activate immune responses. Therefore, the duodenum contains a higher number of $B$ lymphocytes which are stimulated by antigens and involved in humoral immunity.

IgA- and IgG-positive cells are important immunoglobulin secretory cells. Yasuda et al. [27] reported the distribution and quantity of IgA and IgG have been reported to be directly related to the antibody secretion level, and thus reflect the local cellular immune function, of the small intestinal mucosa. In the present study, a greater number of plasmocytes were found in the base of the crypt than in the villi and upper crypt. This indicates that the base of the crypt is a potential site of $B$ lymphocyte terminal differentiation into plasma cells. In both the epithelium and lamina propria, the total number of IgA- and IgG-positive plasma cells was higher in the duodenum than in the jejunum and ileum of newborn yaks. These results are in general agreement with those found in calves, where the total number of plasmocytes is greatest in the duodenum [27]. Wu et al. [26] reported that, by binding to and entrapping antigens in the mucus layer, IgA and IgG limit their access to the mucosa and promote antigen degradation by enzymes within the lumen. Thus, IgA and IgG may provide a backup system in which antigens that have penetrated the mucosal barrier may be cleared by secretory component-mediated transport in the liver [26]. These findings also reflect that the duodenum is a potential major site of effector function for plasma B lymphocytes. It was likely related to duodenal antigens, bile salts, pancreatic secretions, and other local factors that stimulate the production and maturation of antibody-secreting cells.

CD68 is widely used as a marker for intestinal macrophages [16]. Muller reported that, in non-inflamed intestinal mucosa, the lamina propria extracellular matrix releases transforming growth factor beta and interleukin-8, which then recruit blood monocytes to the lamina propria to become resident macrophages [16]. Farache et al. [5] previously showed that functions of intestinal macrophages include antigen presentation, phagocytosis, and production of immune-regulatory factors. In the present study, CD68-positive macrophages were observed in the epithelium. Mowat reported that the main function of most lamina propria macrophages is to phagocytose bacteria (both commensals and pathogens) crossing the epithelium without evoking a strong inflammatory response [15]. Thus, we speculated that epithelial macrophages in the small intestine of yaks may capture and present antigens to lymphocytes, and may also be engaged in the phagocytosis of senescent intestinal epithelial cells. The number of CD68-positive macrophages in the lamina propria was higher in the villi than in the other regions of newborn yaks. Similarly, Bain and Schridde [1] reported that macrophage concentrations were highest in the villi of the intestinal lamina propria. This result suggests that the villi may play a larger role in antigen stimulation and macrophage recruitment than other areas of the lamina propria. Interestingly, macrophages positive for $\mathrm{CD} 68$ were more commonly found in the epithelium and the lamina propria of the ileum than in other regions of the intestine. This suggests that the ileal mucosa of newborn yaks may be more susceptible to antigen capture and processing than the mucosa of the duodenum and jejunum.

Dendritic cells express SIRP $\alpha$ [2]. Mucosal dendritic cells are a key link between innate and acquired 
immunity via their roles in antigen presentation and regulation of T cell activation [2]. In the present study, $\operatorname{SIRP} \alpha$-positive dendritic cells were observed in the epithelium of the small intestine of newborn yaks. It has been previously suggested that dendritic cells can extend their dendrites into the lumen between epithelial cells to handle antigens. In this study, we found a higher number of SIRP $\alpha$-positive cells in the villi of the lamina propria. We believe the appearance of abundant SIRP $\alpha$-positive cells in the villi of the lamina propria was because the site is easily exposed to lymphocytes and bacterial or dietary antigens. Furthermore, we found that SIRP $\alpha$-positive cell concentrations were higher in the epithelium and lamina propria of the ileum and jejunum of newborn yaks. The distribution trend was similar to that for T lymphocytes. Coombes and Powrie [3] showed that dendritic cells expressing the chemokine receptor CCR6 could activate T lymphocytes in response to bacterial invasion. Previous reports have indicated that the wide distribution of dendritic cells in the ileum and jejunum play critical roles in the regulation of intestinal immunity, antigen uptake, and T lymphocyte activation $[3,11,12]$.

In this study, RT-qPCR was used to detect specific immune cell markers. The mRNA expression levels of $\mathrm{CD} 3 \varepsilon, \mathrm{CD} 68$, and $\mathrm{SIRP} \alpha$ were significantly higher than those of $C D 79 \alpha, \lg A$, and $\lg G$ in the same intestinal regions. Thus, we speculate that in the small intestine of newborn yaks, $\mathrm{T}$ lymphocytes, macrophages, and dendritic cells may be more abundant than B lymphocytes and plasmocytes. Additionally, we also found that the mRNA expression levels of $C D 3 \varepsilon, C D 68$, and SIRP $\alpha$ were higher in the ileum, whereas $C D 79 \alpha, \lg A$, and IgG mRNA expression levels were higher in the duodenum. These observations indicate that local humoral immunity may occur more commonly in the ileum, while cellular immunity may occur more commonly in the duodenum.

\section{CONCLUSIONS}

In summary, we immunohistochemically characterised immune cells in the small intestinal mucosa and analysed the mRNA expression level of immune factors in the small intestine of newborn yaks. In the epithelium $\mathrm{CD} 3 \varepsilon$-positive $\mathrm{T}$ lymphocytes were more popular than the other immune cells. In the lamina propria, CD3e-positive T lymphocytes, CD68-positive macrophages and $\operatorname{SIRP} \alpha$-positive dendritic cells were more highly concentrated in the villi, while CD79 $\alpha$-positive B lymphocytes, and IgA- and IgG-pos- itive plasmocytes were more prevalent in the base of the crypt. Furthermore, a higher number of T lymphocytes, macrophages and dendritic cells were located in the epithelium and lamina propria of the ileum than those of the duodenum and jejunum; $B$ lymphocytes, and IgA and IgG plasmocytes were more likely to be observed in the epithelium and lamina propria of the duodenum of newborn yaks than those of the jejunum and ileum. These findings suggest that cellular immunity and antigen presentation are more readily activated in the epithelium, the villi of the lamina propria, and the ileal mucosa of the small intestine of newborn yaks, while humoral immune cells are mostly concentrated in the base of the crypt of the lamina propria and duodenal mucosa. The results from this study will provide information on the baseline characteristics of immune cells in the small intestine of newborn yaks and serve as a reference for future studies on various immunologic reactions in both healthy yaks and those with digestive diseases.

\section{Acknowledgements}

This work was supported by the National Natural Science Foundation of China (Grant No. 32002241\&31772691), Gansu Youth Science and Technology Fund Project (Grant No. 20JR5RA004), Special funds for discipline construction, Gansu Agricultural University (Grant No. GSAU-XKJS2018064), Sheng Tongsheng Innovation Funds, Gansu Agricultural University (Grant No. GSAU-STS1730), and Scientific Research Start-up Funds for Openly-Recruited Doctors, Gansu Agricultural University (Grant No. GSAU-RCZX201703).

\section{Conflict of interest: None declared}

\section{REFERENCES}

1. Bain CC, Schridde A. Origin, differentiation, and function of intestinal macrophages. Front Immunol. 2018; 9: 2733, doi: 10.3389/fimmu.2018.02733, indexed in Pubmed: 30538701.

2. Bekiaris V, Persson EK, Agace WW. Intestinal dendritic cells in the regulation of mucosal immunity. Immunol Rev. 2014; 260(1): 86-101, doi: 10.1111/imr.12194, indexed in Pubmed: 24942684.

3. Coombes JL, Powrie F. Dendritic cells in intestinal immune regulation. Nat Rev Immunol. 2008; 8(6): 435-446, doi: 10.1038/nri2335, indexed in Pubmed: 18500229.

4. Corpa JM, Juste RA, García Marín JF, et al. Distribution of lymphocyte subsets in the small intestine lymphoid tissue of 1-month-old lambs. Anat Histol Embryol. 2001; 30(2): 121-127, indexed in Pubmed: 11379367.

5. Farache J, Zigmond E, Shakhar G, et al. Contributions of dendritic cells and macrophages to intestinal homeostasis and immune defense. Immunol Cell Biol. 2013; 91(3): 
232-239, doi: 10.1038/icb.2012.79, indexed in Pubmed: 23399695.

6. Fries PN, Popowych YI, Guan LeL, et al. Age-related changes in the distribution and frequency of myeloid and $T$ cell populations in the small intestine of calves. Cell Immunol. 2011; 271(2): 428-437, doi: 10.1016/j. cellimm.2011.08.012, indexed in Pubmed: 21917242.

7. German AJ, Hall EJ, Day MJ. Analysis of leucocyte subsets in the canine intestine. J Comp Pathol. 1999; 120(2): 129-145, doi: 10.1053/jcpa.1998.0262, indexed in Pubmed: 10087487.

8. Hitotsumatsu $\mathrm{O}$, Hamada $\mathrm{H}$, Naganuma $\mathrm{M}$, et al. Identification and characterization of novel gut-associated lymphoid tissues in rat small intestine. J Gastroenterol. 2005; 40(10): 956-963, doi: 10.1007/s00535-005-1679-8, indexed in Pubmed: 16261432.

9. Hou HW, Wen Y. Investigation and control of diarrhea in yak calf in Gannan alpine grazing area. Chin J Vet Med. 2007; 43(3): 26, doi: CNKI:SUN:ZSYZ.0.2007-03-012.

10. Hu MD, Jia L, Edelblum KL. Policing the intestinal epithelial barrier: Innate immune functions of intraepithelial lymphocytes. Curr Pathobiol Rep. 2018; 6(1): 35-46, indexed in Pubmed: 29755893.

11. Iwasaki A. Mucosal Dendritic Cells. Ann Rev Immunol. 2007; 25(1): 381-418, doi: 10.1146/annurev.immunol.25.022106.141634.

12. Johansson C, Kelsall BL. Phenotype and function of intestinal dendritic cells. Semin Immunol. 2005; 17(4): 284-294, doi: 10.1016/j.smim.2005.05.010, indexed in Pubmed: 15978836.

13. Korkmaz D, Kum S. A histological and histochemical study of the small intestine of the dromedary camel (Camelus dromedarius). J Camel Pract Res. 2016; 23(1): 111, doi: 10.5958/2277-8934.2016.00018.7.

14. Ma H, Tao W, Zhu S. T lymphocytes in the intestinal mucosa: defense and tolerance. Cell Mol Immunol. 2019; 16(3): 216-224, doi: 10.1038/s41423-019-0208-2, indexed in Pubmed: 30787416.

15. Mowat AM, Bain CC. Mucosal macrophages in intestinal homeostasis and inflammation. J Innate Immun. 2011; 3(6): 550-564, doi: 10.1159/000329099, indexed in Pubmed: 22025201.

16. Muller PA, Matheis F, Mucida D. Gut macrophages: key players in intestinal immunity and tissue physiology. Curr Opin Immunol. 2020; 62: 54-61, doi: 10.1016/j. coi.2019.11.011, indexed in Pubmed: 31841704.

17. Pérez-Cano FJ, Castellote C, González-Castro AM, et al. Developmental changes in intraepithelial $T$ lymphocytes and NK cells in the small intestine of neonatal rats. Pediatr Res. 2005; 58(5): 885-891, doi: 10.1203/01.pdr.0000182187.88505.49, indexed in Pubmed: 16257927.

18. Powell N, MacDonald TT. Recent advances in gut immunology. Parasite Immunol. 2017; 39(6), doi: 10.1111/ pim.12430, indexed in Pubmed: 28370104.

19. Qiu Q, Zhang G, Ma T, et al. The yak genome and adaptation to life at high altitude. Nat Genet. 2012; 44(8): 946-949, doi: 10.1038/ng.2343, indexed in Pubmed: 22751099.

20. Ratcliffe M. B cell development in gut associated lymphoid tissues. Vet Immunol Immunopathol. 2002; 87(3-4): 337-340, doi: 10.1016/s0165-2427(02)00061-2.

21. Solano-Aguilar G, Vengroski K, Beshah E, et al. Characterization of lymphocyte subsets from mucosal tissues in neonatal swine. Develop Comp Immunol. 2001; 25(3): 245-263, doi: 10.1016/s0145-305x(00)00053-7.

22. Suzuki H. Age-dependent changes in intraepithelial lymphocytes (IELs) of the small intestine, cecum, and colon from young adult to aged mice. Arch Gerontol Geriatr. 2012; 55(2): 261-270, doi: 10.1016/j.archger.2011.07.009, indexed in Pubmed: 21840070.

23. Tamura A, Soga H, Yaguchi K, et al. Distribution of two types of lymphocytes (intraepithelial and lamina-propria-associated) in the murine small intestine. Cell Tissue Res. 2003; 313(1): 47-53, doi: 10.1007/s00441-003-0706-4, indexed in Pubmed: 12827490.

24. Waly N, Gruffydd-Jones TJ, Stokes CR, et al. The distribution of leucocyte subsets in the small intestine of healthy cats. J Comp Pathol. 2001; 124(2-3): 172-182, doi: 10.1053/jcpa.2000.0450, indexed in Pubmed: 11222015.

25. Wang Z, Wang L, Qian T, et al. Chemokines and receptors in intestinal B lymphocytes. J Leukocyte Biol. 2018; 103(5): 807-819, doi: 10.1002/jlb.1 ru0717-299rr.

26. Wu CT, Davis PA, Luketic VA, et al. A review of the physiological and immunological functions of biliary epithelial cells: targets for primary biliary cirrhosis, primary sclerosing cholangitis and drug-induced ductopenias. Clin Dev Immunol. 2004; 11(3-4): 205-213, doi: 10.1080/17402520400004177, indexed in Pubmed: 15559365.

27. Yasuda M, Fujino M, Nasu T, et al. Histological studies on the ontogeny of bovine gut-associated lymphoid tissue: appearance of T cells and development of $\lg \mathrm{G}+$ and $\lg \mathrm{A}+$ cells in lymphoid follicles. Dev Comp Immunol. 2004; 28(4): 357-369, doi: 10.1016/j.dci.2003.09.013, indexed in Pubmed: 14698221.

28. Zhou YJ, Gao J, Yang HM, et al. Morphology and ontogeny of dendritic cells in rats at different development periods. World J Gastroenterol. 2009; 15(10): 1246-1253, doi: 10.3748/wjg.15.1246, indexed in Pubmed: 19291826. 